\title{
Effects of multiplication practice on product verification: Integrated structures model or retrieval-induced forgetting?
}

\author{
THOMAS L. PHENIX and JAMIE I. D. CAMPBELL \\ University of Saskatchewan, Saskatoon, Saskatchewan, Canada
}

\begin{abstract}
In the integrated structures model of simple multiplication (Manly \& Spoehr, 1999), it is proposed that retrieval of a multiplication fact (e.g., $2 \times 7=$ ?) activates and strengthens operand multiples representations (e.g., 4, 6, 8, etc.; 14, 21, 28, etc.). In contrast, in the phenomenon of retrieval-induced forgetting (RIF; M. C. Anderson, Bjork, \& Bjork, 1994), it is suggested that operand multiples ought to be suppressed with practice. Participants $(N=72)$ performed 40 blocks of practice trials in which they generated answers to a subset of simple multiplication problems. We then measured response times and errors in a true-false product verification task. Both true and related false equations with practiced operand multiples as presented products were solved relativelypoorly in the verification task. In agreement with RIF, this suggests that operand multiples were suppressed, rather than strengthened, during the practice phase.
\end{abstract}

Manly and Spoehr (1999) proposed the integrated structures model (ISM) of multiplication fact representation. According to the ISM, multiplication problems activate three distinct representations in memory: a whole facts representation (e.g., $2 \times 7=14$ ), an analog code representing the approximate magnitude of the product, and an operand multiples representation consisting of the multiples of the problems operands (e.g., $2 \times 7=14$ activates the operand multiples of $2,4,6,8, \ldots$ and $7,14,21,28 \ldots)$. The three codes are activated simultaneously, and collectively they mediate retrieval of multiplication facts.

In this article, we will focus on the evidence presented by Manly and Spoehr(1999) in connection with the operand multiples representation. Specifically, it is assumed in the ISM that the operand multiples representation is strengthened when a problem (e.g., $4 \times 5=20$ ) is practiced and that this leads to increased interference when the strengthened multiples are tested later in a true-false verification task. For example, deciding that $4 \times 6=16$ is false should be more difficult following practice on $4 \times 5$ because the 4-multiple 16 would be strengthened during practice and become a stronger lure in the context of a false equation. Their experiments appeared to confirm this prediction.

Although Manly and Spoehr's (1999) results supported ISM predictions regarding operand multiples, their results are surprising when viewed from a different perspective.

This research was supported by a grant to the second author from the Natural Sciences and Engineering Research Council of Canada. We gratefully acknowledge Charlotte Manly for her correspondence and provided stimuli. Correspondence concerning this article should be addressed to T. Phenix, Department of Psychology, University of Saskatchewan, 9 Campus Drive, Saskatoon, SK, S7N 5 A5 Canada (e-mail: tom.phenix@usask.ca).
Specifically, Manly and Spoehr's practice-test multiplication paradigm is conceptually quite similar to the paradigms developed by M. C. Anderson and colleagues (M. C. Anderson, Bjork, \& Bjork, 1994; M. C. Anderson \& McCulloch, 1999) to investigate retrieval-induced forgetting (RIF). Specifically, M. C. Anderson and colleagues have shown that repeated cued-retrieval practice on a subset of previously studied items interferes with cued retrieval of semantically related unpracticed items. Thus, in contrast to the predictions of the ISM, the RIF effect suggests that repeatedly practicing specific facts should lead to the suppression of related facts, rather than to the strengthening of them. In the present article, we present evidence that practicing multiplicationfacts does produce RIF and that Manly and Spoehr's evidence for the strengthening of related facts occurred because of a confounding of opposing factors in their stimuli. We will begin by reviewing Manly and Spoehr's paradigm in some detail, and then we will turn to the rationale and design of our experiment.

\section{Manly and Spoehr's (1999) Test of the Operand Multiples Assumption}

To test their assumptions regarding operand multiples representations, Manly and Spoehr (1999) used a multiplication verification task (e.g., $2 \times 7=21$, true or false?). Their main interest was a well-established phenomenon called the lure effect (Campbell \& Tarling, 1996; Stazyk, Ashcraft, \& Hamann, 1982; Winkelman \& Schmidt, 1974): When a presented false answer is a multiple of one of the problem's operands (a related lure such as $2 \times 7=$ 21 ), it is more difficult to reject than a false answer that is not a multiple (an unrelated lure such as $2 \times 7=15$ ). The lure effect, which is the performance difference between these conditions, is typically on the order of 50 to $100 \mathrm{msec}$. 
Related lures are difficult to reject, presumably because they have a strong correct association with one or both of the operands in the context of another problem (e.g., 21 is related to 7 in the context of $3 \times 7=21$ ). To make their true-false decisions, participants can judge the strength of activation produced by the equation or the presented answer. The stronger the activation, the more likely it is that the equation is correct. Correct equations usually produce quite strong activation, but a false equation with a related lure generates stronger activation than an equation with an unrelated lure does. The stronger activation produced by related than by unrelated lures produces the lure effect.

Manly and Spoehr (1999) attempted to manipulate the lure effect in order to provide evidence that practicing multiplication problems would strengthen the operand multiples representation. To do this they tested three sets of eight simple multiplication problems in an experiment with three phases: a verification pretest in which all three sets were tested, a practice phase in which one set was tested repeatedly in a production task (i.e., participants produced the answer to each problem), and a verification posttest during which all three sets were tested again. The practice problem set of eight problems was based on combinations of four operands, either $2 / 5 / 7 / 8$ or $3 / 4 / 6 / 9$, counterbalanced across participants. A set of eight problems based on the unpracticed operands constituted the unrelated problem set. The third set was composed of problems with one practiced operand and one unpracticed operand and was referred to as the mixed problem set.

Manly and Spoehr (1999) proposed that repeatedly solving problems involving a specific subset of operands (i.e., the practice set) would strengthen multiples associated with the practiced operands, whereas multiples of the unpracticed operands would not be strengthened. This procedure should have produced differences in the lure effect in the verification posttest. For the practice set, multiples related to either operand should have produced an increased lure effect in the verification posttest, relative to that in the pretest. For problems in the mixed set, sometimes the related lure was a multiple of the practiced operand and sometimes it was a multiple of the unpracticed operand. Therefore, for the mixed set, Manly and Spoehr (1999) expected the lure effect to be somewhat increased in the posttest but not as much as for the practice set. For the unrelated set, the multiples strengthened during practice were not related to either operand, so they did not expect an increased lure effect for these items. Manly and Spoehr obtained evidence generally supportive of these predictions. Specifically, response time (RT) analyses indicated that in the posttest the largest lure effect was obtained with the practiced problem set, in comparison with the mixed and unrelated problem sets.

\section{A Confound in Manly and Spoehr's (1999) Stimuli?}

Although Manly and Spoehr's (1999) results were anticipated by the ISM, they are surprising given evidence for RIF in similar paradigms. M. C. Anderson et al. (1994) demonstrated that retrieval practice can reduce the accessibility of related associations. Specifically, instead of predicting that related facts would be strengthened with practice, RIF theory predicts that closely related nonretrieved memories should be suppressed. Given this, in Manly and Spoehr's experiment we would have expected practiced operand multiples to be inhibited during practice, resulting in a reduced lure effect for multiples of practiced operands. Thus, the ISM and RIF theories make precisely opposite predictions.

The theoretical contradiction may be resolved by examining a potential confound in the stimuli used by Manly and Spoehr (1999). Specifically, some of the related lures used to represent practiced operand multiples in the verification task also were correct answers to practiced problems. For example, $8 \times 2$ was a problem in the practice set, and its correct answer, 16, was used as the related lure for the practice problem $2 \times 7$ in the verification task (i.e., $2 \times 7=16$ ). For these cases, the stimulus's being a multiple of a practiced operand was confounded with its being the correct answer to a practiced problem. The strengthening of the association between an operand and an answer in the context of one problem (e.g., repeatedly practicing $8 \times 2=16$ ) could make it more difficult to reject that answer when it appears later in combination with one of the practiced operands in the context of a different problem $(2 \times 7=16$, true or false?). In Manly and Spoehr's experiment, this situation existed for some problems in the practiced and the mixed sets but not for problems in the unrelated set, because correct answers to the practice set could never be related lures for the unrelated set. It is possible, therefore, that the practice-related interference they observed was caused by the strengthening of correct associations, rather than the strengthening of operand multiples. The present experiment was designed to discriminate effects associated with multiples of practiced operands from effects caused by practicing correct associations.

The present experiment was conceptually very similar to Manly and Spoehr's (1999) study, but introducing the distinction between practiced operands and practiced answers led us to adopt a new terminology to reduce potential confusion. We will refer to the practice set as the twooperand set, because both operands in each problem were practiced during the production task. The mixed set will be referred to as the one-operand set, because only one of the operands from each problem was practiced. The unrelated set we will call the zero-operand set, because neither operand was practiced during the production task. We will reserve the phrase practiced product to refer to whether or not a lure was a correct answer in the two-operand set. Thus, related lures could be either practiced products (i.e., correct products to problems in the two-operand set) or unpracticed product-related lures. Similarly, unrelated lures can be either practiced or unpracticed product-unrelated lures.

Finally, our experiment used a practice-test design (see Rickard, Healy, \& Bourne, 1994) rather than the pretestpractice-posttest design used by Manly and Spoehr(1999). 
The pretest phase, in which all problems and operands are practiced, would tend to water down the effects of differential practice that we wanted to measure. Eliminating the pretest was intended to maximize our sensitivity to detect practice-related effects during the postpractice verification test. The predictions were straightforward. When lure effects for unpracticed lures are examined, they should, according to the ISM's prediction, decrease from the twooperand to the one-operand to the zero-operand set. The RIF theory predicts the opposite pattern. If a practiced answer confound contributed to Manly and Spoehr's results, we would expect to observe large lure effects with practiced, relative to unpracticed, product lures.

\section{METHOD}

\section{Participants}

Seventy-two first-year psychology students (21 men, 51 women) from the University of Saskatchewan received course credit for their participation. Ages ranged from 18 to 35 years $(M=21)$.

\section{Stimuli and Design}

We used the same three sets of eight simple multiplication problems that were used by Manly and Spoehr (1999; see Appendix A). One set of eight problems used the operands $2,5,7$, and 8 , and another set used the operands $3,4,6$, and 9 . One or the other of these two sets (referred to as the two-operand set) was tested in 40 blocks in a production task prior to the verification task. The third set of eight was composed of problems that used one operand from each of the $2 / 5 / 7 / 8$ and the 3/4/6/9 sets. We called these problems the one-operand set because each problem included exactly one operand that had appeared in the two-operand set. The unpracticed set (either the $2 / 5 / 7 / 8$ or the $3 / 4 / 6 / 9$ set) was called the zero-operand set, because neither of their operands had appeared in the two-operand set. The 2/5/7/8 set was the two-operand set for odd-numbered participants, and the 3/4/6/9 set was the two-operand set for even-numbered participants.

After the two-operand set was practiced in the production task, all 24 problems in the three sets were tested in each of six verification blocks. Across the six blocks each problem was tested twice in a true equation (e.g., $2 \times 7=14$ ) and with four types of false lures. There were eight true equations in each block, and the two true equations for each problem were separated by three blocks. Across blocks, the four lure conditions for each problem were randomly interleaved with the true equations with the constraint that, across problems, all four lure conditions were equally likely to precede or follow true equation trials.

The four types of false lures included practiced product-related lures, practiced product-unrelated lures, unpracticed product-related lures, and unpracticed product-unrelated lures. A practiced productrelated lure was a correct answer to a problem in the two-operand set (i.e., a practiced answer) and a multiple of an operand in the verification problem (i.e., related). A practiced product-unrelated lure was a correct answer to a two-operand problem (i.e., practiced) but not a multiple of either operand in the verification problem (i.e., unrelated). An unpracticed product-related lure was not a correct answer to any problem in the two-operand set (i.e., an unpracticed answer) but was a multiple of an operand in the verification problem (i.e., related). Finally, an unpracticed product-unrelated lure was not a correct answer to a two-operand set problem (i.e., unpracticed) and also not a multiple of either operand in the verification problem (i.e., unrelated).

Appendices A and B present the lures used for each problem in the $2 / 5 / 7 / 8$ and the $3 / 4 / 6 / 9$ sets, respectively. For each problem we generated all possible lures of each type by using the same two constraints that were used by Manly and Spoehr (1999): (1) Lures were a correct answer to a simple multiplication problem in the range from $2 \times 2$ to $9 \times 9$, and (2) the absolute numerical distance be- tween the lure and the correct answer was less than or equal to two times the larger operand.

As the appendices show, the number of lures of each type that met all the requirements for a given problem varied from as many as seven to as few as zero (e.g., if $2 \times 5$ was a two-operand set problem there was no lure that met all the requirements for a practiced product-unrelated lure). Similarly, because the zero-operand and two-operand sets had no common operands it was not possible for a correct answer to a two-operand problem to be operand related to a zero-operand problem; therefore, practiced product-related lures are not defined for the zero-operand set. When no lure was available for a problem in a given condition, a randomly selected prime number less than 100 was presented and that trial was excluded from the analyses. Otherwise, one of each type of lure for each problem was selected at random from among those available.

\section{Procedure}

The participants performed the half-hour experiment in a quiet room with the experimenter present. An IBM-type computer was used with two high-resolution VGA monitors that displayed the stimuli. The experimenter and the participant viewed separate monitors. The participant sat at a comfortable viewing distance from the monitor and wore a lapel microphone that triggered a soundactivated relay switch connected to the computer's parallel port. The relay switch provided a signal that stopped a software clock, which was accurate to $\pm 1 \mathrm{msec}$.

The basic design of the study was explained, and the participant was instructed to respond quickly but accurately. The experiment began with a production task, which consisted of 40 blocks of the eight problems in the two-operand set. For the production task, the participant was required to state the correct answer aloud. In each block the eight problems were presented in random order without replacement. The blocks were continuous, but the participants received a brief rest after 20 blocks. The experimenter initiated each set of 20 blocks by pressing the space bar.

A 1-sec central fixation dot preceded each production trial. The problem then appeared in horizontal orientation with the times sign at fixation. The problems appeared in a seven-character field (e.g., $3 \times 8=$ ?) in white characters against a dark background. Each character space was approximately $3 \mathrm{~mm}$ wide and $5 \mathrm{~mm}$ high. Timing began with presentation of the problem (synchronized with the raster scan), and the problem remained on the screen until a sound triggered the sound-activated relay and stopped the timer. Triggering the relay caused the problem to disappear immediately from the screen, which allowed the experimenter to detect and mark RTs that were spoiled by failures of the voice key. Once the experimenter entered the participant's response, the fixation dot appeared for the next trial. The participants received no feedback about speed or accuracy during production trials.

Verification trials immediately followed completion of the production task. Before each of the six verification blocks, the following information appeared on the screen: "The following is a True/ False block. Please say aloud whether each problem is either TRUE or FALSE." The experimenter initiated each block by pressing the space bar. The participants received all 24 problems (i.e., the twooperand, one-operand, and zero-operand sets) in a random order in each block. Verification equations consisted of the problem and a presented true or false answer (e.g., $4 \times 8=32$ or $3 \times 5=25$ ). Speed-accuracy instructions, stimulus presentation, and response timing were the same as that for the production task. The participants received no feedback about speed or accuracy during verification trials.

\section{RESULTS}

The $1.6 \%$ of the correct RTs that were more than 2.5 standard deviations from each participant's mean per con- 
dition were discarded as outliers (Van Selst \& Jolicœur, 1994), and another $3.6 \%$ were discarded as spoiled because of failures of the voice key. The overall error rate was $3.6 \%$. Table 1 presents the mean RTs for true equations and for equations with related and unrelated lures. The means for related and unrelated lures are also presented in this table, including the combined practiced product and unpracticed product lures, the unpracticed product lures only, and practiced product lures only. Table 2 presents the corresponding means for error rates.

The analyses are organized into five sections. To demonstrate that the experiment effectively replicated the lure effects observed by Manly and Spoehr (1999), we will examine the lure effects of combined practiced product and unpracticed product lures in the first section. In the second section, we will examine the lure effects of unpracticed product lures only. This provides a test of the contrasting predictions of the ISM and RIF theories that is uncontaminated by the potential effects of practiced answers. In the third section, we will examine the differential effects of practiced product and unpracticed product lures. In the fourth section, we will examine predictions concerning true equations. In the final section, we will compare lure effects for multiples that, theoretically, have or have not been activated.

\section{Overall Lure Effects as a Function of Problem Set}

To determine whether lure effects varied with practice, as was predicted by Manly and Spoehr's (1999) ISM, we analyzed mean RT and percentage of errors as a function of lure (related or unrelated) and set (two-operand, one-

Table 1

Means and Standard Deviations for Response Time (in Milliseconds) for True Equations, Practiced and Unpracticed

Product Lures Combined, Unpracticed Product Lures Only, and Practiced Product Lures Only as a Function of Set

Set

\begin{tabular}{|c|c|c|c|c|c|c|}
\hline \multirow[b]{3}{*}{ Lure Type } & & & & & & \\
\hline & \multicolumn{2}{|c|}{ Two-Operand } & \multicolumn{2}{|c|}{ One-Operand } & \multicolumn{2}{|c|}{$\overline{\text { Zero-Operand }}$} \\
\hline & $M$ & $S D$ & $M$ & $S D$ & $M$ & $S D$ \\
\hline \multicolumn{7}{|c|}{ True Equations } \\
\hline & 98 & 164 & 1,138 & 208 & 1,052 & 174 \\
\hline
\end{tabular}

\begin{tabular}{|c|c|c|c|c|c|c|}
\hline \multicolumn{7}{|c|}{ Unpracticed and Practiced Product Lures Combined } \\
\hline Related & 1,193 & 205 & 1,248 & 230 & 1,179 & 235 \\
\hline Unrelated & 1,095 & 193 & 1,196 & 263 & 1,147 & 225 \\
\hline Lure effect & \multicolumn{2}{|c|}{98} & \multicolumn{2}{|c|}{52} & \multicolumn{2}{|c|}{32} \\
\hline \multicolumn{7}{|c|}{ Unpracticed Product Lures Only } \\
\hline Related & 1,152 & 200 & 1,247 & 226 & 1,179 & 235 \\
\hline Unrelated & 1,127 & 200 & 1,125 & 255 & 1,144 & 243 \\
\hline Lure effect & \multicolumn{2}{|c|}{25} & \multicolumn{2}{|c|}{122} & \multicolumn{2}{|c|}{35} \\
\hline \multicolumn{7}{|c|}{ Practiced Product Lures Only } \\
\hline Related & 1,235 & 243 & 1,249 & 269 & - & \\
\hline Unrelated & 1,063 & 214 & 1,266 & 323 & 1,150 & 240 \\
\hline Lure effect & \multicolumn{2}{|c|}{172} & \multicolumn{2}{|c|}{-17} & & \\
\hline
\end{tabular}

Note-Dashes indicate that related lures were not definable for that condition. Lure effect $=$ related minus unrelated lures.
Table 2

Means and Standard Deviations for Percentage Error Rates for True Equations, Practiced and Unpracticed Product Lures Combined, Unpracticed Product Lures Only, and Practiced Product Lures Only as a Function of Set

\begin{tabular}{|c|c|c|c|c|c|c|}
\hline \multirow[b]{3}{*}{ Lure Type } & \multicolumn{6}{|c|}{ Set } \\
\hline & \multicolumn{2}{|c|}{ Two-Operand } & \multicolumn{2}{|c|}{ One-Operand } & \multicolumn{2}{|c|}{ Zero-Operand } \\
\hline & $M$ & $S D$ & $M$ & $S D$ & $M$ & $S D$ \\
\hline \multicolumn{7}{|c|}{ True Equations } \\
\hline & 2.2 & 3.8 & 5.8 & 6.8 & 3.1 & 4.7 \\
\hline \multicolumn{7}{|c|}{ Practiced and Unpracticed Product Lures Combined } \\
\hline Related & 4.5 & 5.9 & 8.2 & 8.3 & 7.1 & 9.8 \\
\hline Unrelated & 2.0 & 4.3 & 1.1 & 4.5 & 3.2 & 5.1 \\
\hline Lure effect & \multicolumn{2}{|c|}{2.5} & \multicolumn{2}{|c|}{7.1} & \multicolumn{2}{|c|}{3.9} \\
\hline \multicolumn{7}{|c|}{ Unpracticed Product Lures Only } \\
\hline Related & 3.0 & 5.7 & 6.6 & 8.9 & 7.1 & 9.8 \\
\hline Unrelated & 3.1 & 6.7 & 0.2 & 1.7 & 2.6 & 7.2 \\
\hline Lure effect & \multicolumn{2}{|c|}{-0.1} & \multicolumn{2}{|c|}{6.4} & \multicolumn{2}{|c|}{4.5} \\
\hline
\end{tabular}

Practiced Product Lures Only

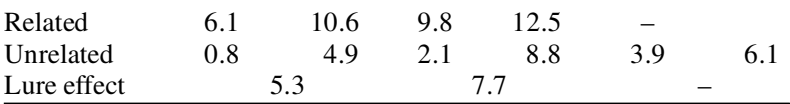

Note-Dashes indicate that related lures were not definable for that condition. Lure effect $=$ related minus unrelated lures.

operand, or zero-operand). According to Manly and Spoehr, the ISM predicts that practicing the two-operand set during the production task should result in larger lure effects for the two-operand set, relative to the one-operand and zero-operand sets.

Response times. The experiment produced the standard lure effect, with longer mean RTs for related lures $(1,207 \mathrm{msec})$ than for unrelated lures $(1,146 \mathrm{msec})$ $\left[F(1,71)=46.71, M S_{\mathrm{e}}=8,591, p<.001\right]$. Overall, RTs also differed across the three problem sets $[F(2,142)=$ $\left.19.76, M S_{\mathrm{e}}=11,953, p<.001\right]$ : Mean RTs were greater overall for the one-operand set $(1,222 \mathrm{msec})$ than for either the two-operand $(1,144 \mathrm{msec})$ or the zero-operand $(1,163 \mathrm{msec})$ sets. These main effects were qualified by the lure $\times$ set interaction $\left[F(2,142)=5.31, M S_{\mathrm{e}}=7,848\right.$, $p=.006]$. Figure 1 presents the mean lure effect and $95 \%$ confidence interval for each problem set. The mean lure effects were 98, 52, and $32 \mathrm{msec}$ for the two-operand, oneoperand, and zero-operand sets, respectively. Thus, the lure $\times$ set interaction in RT was as predicted by the ISM, and it substantively replicates the RT lure effects that were observed by Manly and Spoehr.

Percentage of errors. Although Manly and Spoehr (1999) did not report detailed analyses of errors, the ISM presumably predicts the largest lure effect on errors for the two-operand set, followed by the one-operand and zerooperand sets. Related lures produced more errors $(6.6 \%)$ than did unrelated lures $(2.1 \%)\left[F(1,71)=48.1, M S_{\mathrm{e}}=\right.$ $45.8, p<.001]$. The two-operand set produced slightly fewer errors $(3.2 \%)$ than the one-operand $(4.7 \%)$ and zero-operand $(5.2 \%)$ sets did $\left[F(2,142)=4.09, M S_{\mathrm{e}}=\right.$ $34.4, p=.019]$. As Figure 1 shows, there was also a lure $X$ set interaction that occurred because the lure effect on 

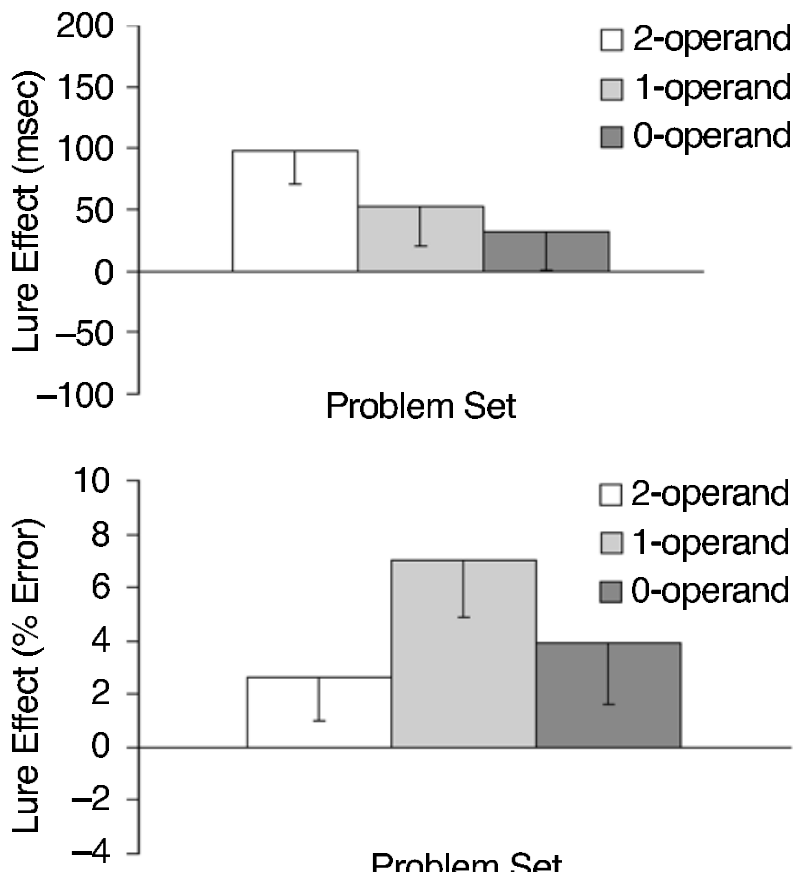

Figure 1. Lure effects in mean response time (RT) and error rate as a function of set (practiced and unpracticed product lures combined).

errors was greater for the one-operand set $(7.1 \%)$ than for either the two-operand set $(2.5 \%)$ or the zero-operand set $(3.9 \%)\left[F(2,142)=5.9, M S_{\mathrm{e}}=31.9, p=.004\right]$. Thus, unlike the pattern of RT results, the pattern of lure effects on errors across the problem sets was not predicted by the ISM.

\section{Lure Effects for Unpracticed Answers}

The analysis of overall lure effects apparently confirmed the RT predictions of the ISM, but that analysis included both unpracticed and practiced answers. As was explained in the introduction, a related lure may be especially difficult to reject if it is also a correct answer to a practiced problem (i.e., a member of the two-operand set). In this case, practiced product lures would contribute to larger lure effects for the two-operand and one-operand sets than for the zero-operand set: By definition, practiced product lures cannot be related lures for the zero-operand set (i.e., the zero-operand and two-operand sets do not share any common operands). A proper test of the ISM's lure effects predictions should examine only unpracticed answers and should exclude practiced answers. Therefore, we analyzed mean RT and percentage of errors as a function of lure (related or unrelated) and set (two-operand, one-operand, or zero-operand) for unpracticed product lures only. The corresponding means for RTs and errors appear in Tables 1 and 2, respectively.

Response times. Mean RTs were longer for related lures $(1,193 \mathrm{msec})$ than for unrelated lures $(1,132 \mathrm{msec})$, which confirmed a lure effect for unpracticed answers
$\left[F(1,71)=34.26, M S_{\mathrm{e}}=11,491, p<.001\right]$. Mean RT was longest for the one-operand problem set $(1,186 \mathrm{msec})$, followed by the zero-operand $(1,162 \mathrm{msec})$ and two-operand $(1,139 \mathrm{msec})$ sets $\left[F(2,142)=5.26, M S_{\mathrm{e}}=14,838, p=\right.$ .006]. Figure 2 illustrates the lure $\times$ set interaction for unpracticed answers $\left[F(2,142)=7.73, M S_{\mathrm{e}}=13,032, p=\right.$ $.001]$. In contrast to the overall analysis of lure effects, wherein the effect was greatest for the two-operand set (see Figure 1), the RT lure effect was nominally smallest for two-operand problems when only unpracticed answers were analyzed; specifically, the lure effect for the oneoperand set $(122 \mathrm{msec})$ was more substantial than that for both the two-operand $(25 \mathrm{msec})$ and zero-operand $(35 \mathrm{msec})$ sets. This implies that the relatively large lure effect for the two-operand set in the overall analysis was caused entirely by practiced product lures. The following analysis of errors confirmed that the lure effect was reduced for the two-operand set when only unpracticed product lures were analyzed.

Percentage of errors. Unpracticed product-related lures $(5.6 \%)$ produced more errors than did unpracticed product-unrelated lures $(1.9 \%)\left[F(1,71)=23.7, M S_{\mathrm{e}}=\right.$ $59.2, p<.001]$. The overall error rates differed slightly across the two-operand (3.0\%), one-operand $(3.4 \%)$, and zero-operand $(4.8 \%)$ sets $\left[F(2,142)=3.75, M S_{\mathrm{e}}=35.0\right.$, $p=.026]$. Most importantly, as Figure 2 illustrates, there was no lure effect observed for the two-operand set $(-0.1 \%)$, whereas the lure effect on errors was substantial for both the one-operand (6.4\%) and zero-operand (4.5\%)
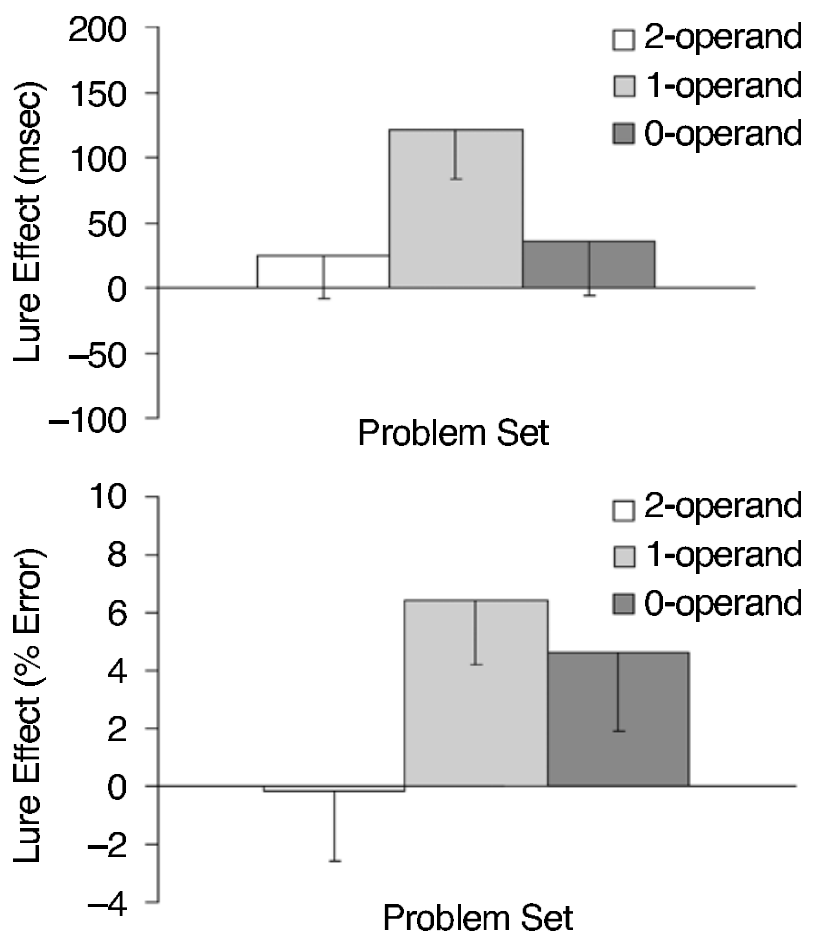

Figure 2. Lure effects for unpracticed product lures in mean response time (RT) and error rate as a function of set. 
sets, giving rise to the lure $\times$ set interaction $[F(2,142)=$ 9.7, $\left.M S_{\mathrm{e}}=42.2, p<.001\right]$.

Taken together, the RT and error analyses of unpracticed product lures demonstrated that the lure effect for the two-operand set, relative to the other two sets, tended to be reduced by practice rather than increased by practice. The results therefore appear to be more consistent with RIF, rather than caused by increased interference, as is predicted by the ISM. The evidence in favor of the ISM in the RT analysis of the overall lure effect (i.e., including both practiced and unpracticed answers) apparently arose entirely in connection with practiced product lures. The following analyses provided a direct comparison of lure effects for practiced product and unpracticed product lures.

\section{Lure Effects for Practiced Versus \\ Unpracticed Lures}

The analysis of lure effects for practiced product versus unpracticed product lures is restricted to the two-operand and one-operand sets, because practiced product-related lures do not exist for the zero-operand set (i.e., that cell is undefined in the design). Therefore, we analyzed mean RT and percentage of errors in $2 \times 2 \times 2$ analyses of variance (ANOVAs), with the factors of lure (related or unrelated), practice (practiced product lures or unpracticed product lures), and problem set (two-operand or oneoperand).

Response times. Mean RTs were longer for related lures $(1,221 \mathrm{msec})$ than for unrelated lures $(1,145 \mathrm{msec})$ $\left[F(1,71)=50.8, M S_{\mathrm{e}}=16,103, p<.001\right]$. Overall, the mean RT was faster for the two-operand problem set $(1,144 \mathrm{msec})$ than that for the one-operand problem set $(1,222 \mathrm{msec})\left[F(1,71)=41.4, M S_{\mathrm{e}}=20,992, p<.001\right]$. Practiced product lures $(1,203 \mathrm{msec})$ led to longer RTs overall than did unpracticed product lures $(1,163 \mathrm{msec})$ $\left[F(1,71)=12.1, M S_{\mathrm{e}}=19,481, p=.001\right]$. These main effects were qualified, however, by the lure $\times$ set $\times$ practice interaction $\left[F(1,71)=33.3, M S_{\mathrm{e}}=21,889, p<.001\right]$. The source of the interaction is apparent in Figure 3, which presents the mean lure effects (and 95\% confidence intervals) as a function of set and lure type (i.e., practiced product vs. unpracticed product). The figure shows that the triple interaction occurred because, for the two-operand set, there was a large lure effect with practiced product lures but no lure effect with unpracticed product lures, whereas the one-operand set presented the opposite pattern (i.e., a lure effect with unpracticed product lures but not with practiced product lures).

Figure 3 confirms that the overall larger RT lure effect for the two-operand set arose entirely from practiced product lures, whereas unpracticed product lures produced no
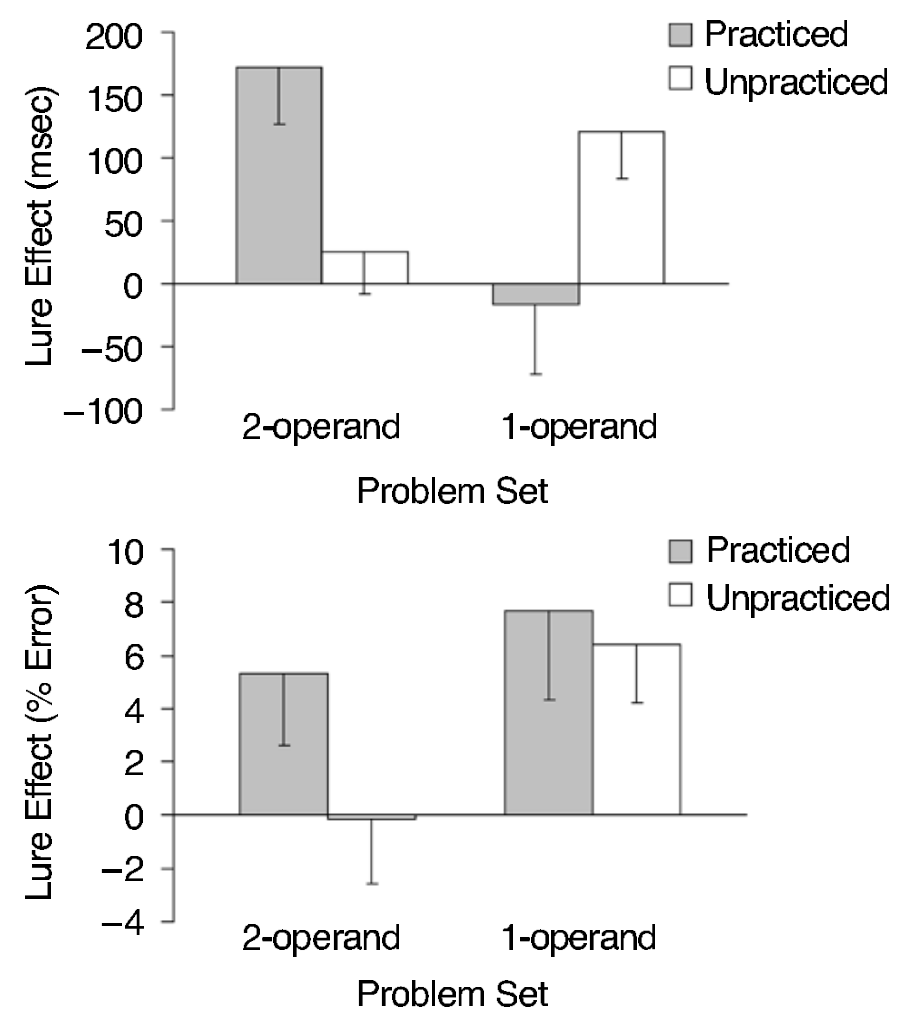

Figure 3. Lure effects in mean response time (RT) and error rate as a function of set and lure type (practiced product vs. unpracticed product lures). 
lure effect for the two-operand set. Interestingly, Table 1 shows that the 172-msec lure effect for practiced product lures with the two-operand set resulted from two distinct effects of practice on related and unrelated lures. First, mean RTs for practiced product-related lures $(1,235 \mathrm{msec})$ was significantly greater than that for unpracticed productrelated lures $(1,152 \mathrm{msec})[t(71)=4.1, S E=20.4, p<$ $.001]$. Second, and in contrast, mean RTs for practiced product-unrelated lures $(1,063 \mathrm{msec})$ was faster than that for unpracticed product-unrelated lures $(1,127 \mathrm{msec})$ $[t(71)=-3.6, S E=17.6, p=.001]$. For unrelated lures, a set (two-operand vs. zero-operand) $\times$ lure (practiced product vs. unpracticed product lure) analysis confirmed a significant interaction, which resulted from faster RTs for practiced product-unrelated lures than for unpracticed product-unrelated lures only for two-operand set problems $\left[F(1,71)=5.7, M S_{\mathrm{e}}=15,338, p=.02\right]$. Thus, the large lure effect for the two-operand set with practiced product lures reflected both slower RTs for practiced product-related lures and faster RTs for practiced product-unrelated lures.

There was no RT lure effect for practiced product lures with the one-operand set (see Figure 3 ). We think that this probably represents a ceiling effect on RTs: As Table 1 shows, the longest mean RT in the experiment was observed with practiced product-unrelated lures tested in the context of the one-operand set $(1,266 \mathrm{msec})$. If the participants adopted an internal RT deadline around this time, we would not be able measure a lure effect in RT because RTs for both related and unrelated lures would encounter the deadline. In this case, we would expect especially high error rates for practiced product-related lures with the one-operand set. Table 2 confirms that this cell produced the highest error rate in the experiment $(9.8 \%)$ ). Furthermore, the following analysis of errors demonstrates that we observed a strong lure effect on errors with the oneoperand set for both practiced product and unpracticed product lures.

Error rates. We observed the standard lure effect, with more errors for related lures $(6.4 \%)$ than for unrelated lures $(1.5 \%)\left[F(1,71)=47.1, M S_{\mathrm{e}}=70.7, p<.001\right]$. There were fewer errors for the two-operand set $(3.2 \%)$ than for the one-operand set $(4.7 \%)\left[F(1,71)=7.0, M S_{\mathrm{e}}=41.1\right.$, $p=.01]$. Errors were more common overall for practiced product lures $(4.7 \%)$ than for unpracticed product lures $(3.2 \%)\left[F(1,71)=4.2, M S_{\mathrm{e}}=76.2, p=.045\right]$. Unlike the results of the RT analysis, the lure $\times$ set $\times$ practice interaction was not significant $\left[F(1,71)=2.9, M S_{\mathrm{e}}=54.4\right.$, $p=.09$ ], but the pattern (see Figure 3 ) reinforces our conclusions from the RT analysis. For the two-operand set, there was a strong lure effect with practiced product lures but no lure effect for unpracticed product lures. In contrast, for the one-operand set, there was a strong lure effect on errors with both practiced product and unpracticed product lures.

With respect to the two-operand set, as in the RT analysis, the greater lure effect in errors with practiced product $(+5.3 \%)$ than with unpracticed product $(-0.1 \%)$ lures reflected two effects. As Table 2 shows, the mean error rate for practiced product-related lures $(6.1 \%)$ was significantly greater than that for unpracticed product-related lures $(3.0 \%)[t(71)=2.2, S E=1.5, p=.003]$. In contrast, the mean error rate for practiced product-unrelated lures $(0.8 \%)$ was lower than that for unpracticed productunrelated lures $(3.1 \%)[t(71)=-2.4, S E=0.9, p=.02]$. A set (two-operand vs. zero-operand) $\times$ lure (practiced product vs. unpracticed product lure) analysis of the unrelated lure condition confirmed the significant interaction $\left[F(1,71)=6.5, M S_{\mathrm{e}}=35.4, p=.01\right]$. Thus, both the RT and error analyses demonstrated that the relatively large lure effect for the two-operand set with practiced product lures reflected both reduced performance for practiced product-related lures and enhanced performance for practiced product-unrelated lures. We will return to these novel effects in the Discussion section.

The foregoing analyses confirmed that the larger overall lure effect for the two-operand set (see Figure 1) arose entirely in connection with practiced product lures. In fact, practicing the two-operand set tended to reduce the lure effect for unpracticed answers, rather than to increase the lure effect, as was predicted by the ISM. Such a reduction in lure effects for unpracticed product lures would be expected if practicing the two-operand set produced RIF of multiples of the practiced problems. In the following analyses, we present additional evidence that RIF had a substantial impact in our experiment.

\section{Performance on True Equations as a Function of Set}

The ISM predicts that practice on the two-operand problem set should strengthen the associated operand multiples representations. This assumption leads to predictions for true, one-operand set equations: The correct answers to one-operand set problems are necessarily among the multiples assumed to be strengthened by the two-operand set. By way of analogy to the ISM's prediction of greater lure effects when false multiples are strengthened, we would expect performance of true equations for the oneoperand problem set to be enhanced when the strengthened multiple is the correct answer. In contrast, RIF theory predicts that multiples of the practiced operands are suppressed. Since correct answers to one-operand problems are among those predicted to be suppressed, RIF theory predicts that performance of true equations for the oneoperand problem set should be reduced by practice on the two-operand set. Once again, the ISM and RIF theories lead to opposite predictions.

To test these predictions, we compared true equation performance for the one-operand set to performance on the zero-operand set. The zero-operand set provides a theoretically appropriate baseline, because it contains no practiced operands and therefore should not be affected either by strengthening effects (in accordance with the ISM) or suppression effects (in accordance with the RIF theory). One possible concern, however, is that any difference might be caused by differences in item-specific difficulty between the sets (i.e., the problems making up 
the two-operand and zero-operand sets were counterbalanced, but the one-operand set was fixed). Therefore, we included unpracticed product-unrelated lures (UUL) in the analysis as a control condition. UUL trials should not be affected by relatedness effects but should be sensitive to item-specific differences in difficulty between the one-operand and zero-operand sets.

Response times. Mean RTs were analyzed in an equation type (true vs. UUL) $\times$ set (two-operand, one-operand, or zero-operand) ANOVA. As is shown in the top panel of Figure 4, there was a type $\times$ set interaction $[F(2,142)=$ 40.7, $\left.M S_{\mathrm{e}}=10,782, p<.001\right]$. This occurred because mean RTs for UUL trials were constant across sets $(1,127$, 1,125 , and $1,144 \mathrm{msec}$ ), whereas RTs for true equations were longest for the one-operand set $(1,138 \mathrm{msec})$, followed by the zero-operand set $(1,052 \mathrm{msec})$ and the twooperand set $(982 \mathrm{msec})$. The relatively fast RTs for the two-operand set would be expected, of course, given that these problems had received substantial practice.

The contrast between the one-operand and zero-operand sets is more theoretically important in the present context. A separate type $\times$ set ANOVA that included only the oneoperand and zero-operand sets confirmed the interaction $\left[F(1,71)=22.25, M S_{\mathrm{e}}=8,831, p<.001\right]$. Specifically, there was no difference in RTs between sets for the UULs, but true equations from the one-operand set had longer mean RTs than did those in the zero-operand set. This is consistent with the expected inhibition effects of practic- ing the two-operand set, which is predicted by RIF theory. The following error analyses reinforce this conclusion.

Error rates. The bottom panel of Figure 4 presents the corresponding error data. The one-operand set yielded more errors $(5.8 \%)$ than either the two-operand $(2.2 \%)$ or the zero-operand $(3.1 \%)$ sets $\operatorname{did}\left[F(2,142)=12.8, M S_{\mathrm{e}}=\right.$ $25.9, p<.001]$. In addition, a higher error rate was observed for the one-operand set than for the zero-operand set $[t(71)=2.8, S E=0.9, p=.006]$. The truth (true equations vs. UUL) $\times$ set (two-operand, one-operand, or zerooperand) interaction $\left[F(2,142)=3.8, M S_{\mathrm{e}}=26.6, p=\right.$ .023 ] occurred because UUL trials for the one-operand set were easier than were the UUL trials from the other two sets, whereas the one-operand set produced more errors on true equations than did the other two sets. The truth $\times$ set interaction was also significant when only one-operand and zero-operand sets were contrasted $[F(1,71)=17.18$, $\left.M S_{\mathrm{e}}=26.6, p<.001\right]$. The UUL trials suggest that the one-operand set was, if anything, easier than the zerooperand set, yet performance of the one-operand set was relatively poor in the context of true equations. Thus, the results are consistent with the interference effect predicted by the RIF theory.

\section{Activated Versus Nonactivated Multiples}

The ISM and RIF theories make additional predictions for the one-operand set regarding multiples that have or have not been activated during practice on the two-operand

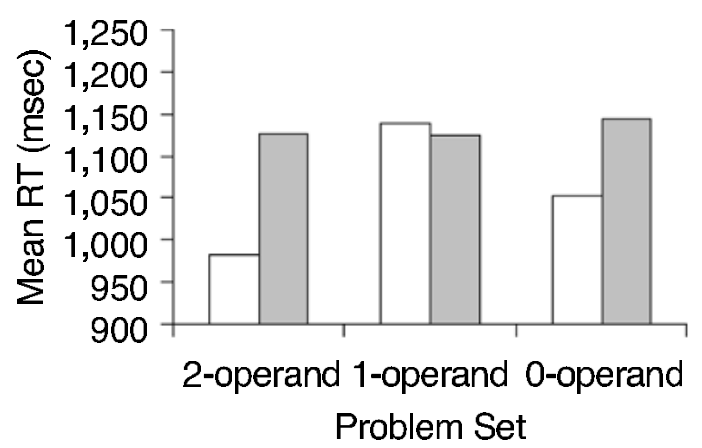

TRUE

Unpracticed

Product-Unrelated

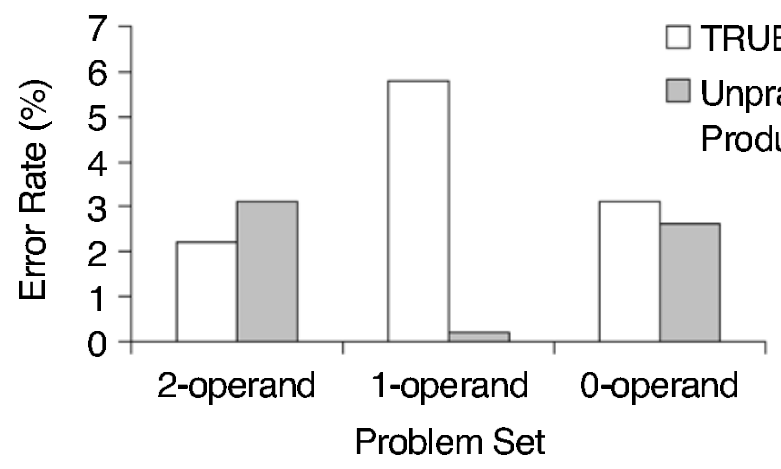

Figure 4. Mean response time (RT) and error rate as a function of set (one-operand vs. zero-operand problems) and equation type (true vs. unpracticed product-unrelated lures). 
set. Problems in the one-operand set, by definition, always have one practiced and one unpracticed operand. Thus, related lures from the one-operand set are either multiples of the practiced operand (activated multiples) or multiples of the unpracticed operand (nonactivated multiples). Note that the practiced operand for the one-operand set is counterbalanced across participants (i.e., it is determined by whether a participant practiced the $3 / 4 / 6 / 9$ or the $2 / 5 / 7 / 8$ problems sets); therefore, the specific answers that serve as activated and nonactivated multiples are also counterbalanced.

The predictions are as follows: According to the ISM, activated multiples would be strengthened during practice on the two-operand problem set, whereas nonactivated multiples would not be directly affected by practice on the two-operand set. Therefore, the ISM predicts slower RTs to be caused by the rejection of activated multiples rather than by the rejection of nonactivated multiples. In contrast, RIF predicts suppression of activated multiples and, therefore, faster RTs to rejected activated multiples than to nonactivated multiples.

An examination of mean RTs for activated and nonactivated multiples revealed that activated multiples $(1,241 \mathrm{msec})$ were nominally faster than nonactivated multiples $(1,268 \mathrm{msec})[t(71)=-1.0, S E M=25.8, p=$ .30]. An examination of errors, however, indicated that activated multiples $(9.0 \%)$ had nominally higher error rates than did nonactivated multiples $(6.7 \%)[t(71)=1.4$, $S E M=1.7, p=.18]$. These results, therefore, provided no conclusive evidence for or against the RIF theory or the ISM when activated and nonactivated multiples from the one-operand set were used.

\section{DISCUSSION}

In the present experiment, we tested predictions that follow from the ISM and RIF theories regarding processes that might affect operand multiples representations. Specifically, we looked for evidence to indicate whether operand multiples are strengthened or weakened by multiplication practice in the production task. The results from the postpractice verification task were similar to the results obtained by Manly and Spoehr (1999) when the analyses included both practiced product and unpracticed product lures. Specifically, the lure effect was largest for the twooperand problem set and smallest for the zero-operand problem baseline. Thus, repeatedly retrieving answers to multiplication problems appeared to strengthen the multiples of practiced operands, which increased the lure effect, as was predicted by the ISM.

Nonetheless, alternative analyses that excluded lures that were correct answers to problems in the two-operand set (i.e., that included only unpracticed answers) indicated a reduced lure effect for the two-operand problem set. Thus, in contrast to the overall analysis, the analysis of unpracticed answers indicated RIF of multiples of practiced operands. A comparison of lure effects for practiced product and unpracticed product lures showed that, for the two- operand set, only practiced product lures produced a large lure effect, whereas unpracticed product lures presented no lure effect. Thus, Manly and Spoehr's (1999) findings apparently resulted from interference caused by the strengthening of correct associations (i.e., practiced productrelated lures) rather than from the strengthening of operand multiples. Instead, lure effects of operand multiples appeared to be weakened by practice, as was predicted by RIF theory.

Analyses based on true equations involving the oneoperand problem set provided additional evidence for RIF and against the ISM hypothesis of operand multiples strengthening. Correct answers to problems in the oneoperand set were always multiples of practiced operands; consequently, the ISM predicted strengthening of these answers and therefore better performance for true, oneoperand set equations than for equations in the zerooperand set. In contrast, the RIF theory predicted reduced true equation performance for the one-operand problem set than for the zero-operand set. Our results clearly favored the latter prediction and indicted relatively poor performance on true equations in the one-operand problem set. Thus, performance on both true and false equations indicated that operand multiples of practiced operands were less accessible after practice, as was expected on the basis of RIF theory.

Although our findings demonstrated RIF, they do not directly support an inhibition theory of RIF. RIF theory (e.g., M. C. Anderson \& Bell, 2001; M. C. Anderson \& Spellman, 1995), proposes suppression of the practiced operand multiples representations caused by an inhibition process; but noninhibitory, activation-based processes could also account for the RIF phenomenon (J. R. Anderson \& Reder, 1999). Our results do not discriminate inhibitory from activation-based theories, but they clearly demonstrate that operand multiples representations are subject to RIF rather than to strengthening as a result of practice.

Although our results contradict Manly and Spoehr's (1999) assumptions about how practice should affect operand multiples, the results nonetheless provide support for the assumption of an operand multiples representation. Specifically, the reduced lure effect observed for the twooperand problem set with unpracticed product lures demonstrates that the operand multiples were covertly processed during multiplication production. This converges with evidence that multiples are often produced as errors (Campbell \& Tarling, 1996) and produce strong false-lure effects in verification. The unique feature of Manly and Spoehr's paradigm, however, is that it allows one to measure aftereffects of multiplication practice on operand multiples that were not consciously processed during practice (i.e., not presented or retrieved). The residual effects appearing in both true and false postpractice verification trials provide the most direct evidence to date that processing of operand multiples is a normal component of multiplication fact retrieval.

Apart from Manly and Spoehr's (1999) ISM model, several other models have proposed an operand multiples rep- 
resentation (see Ashcraft, 1992, for a review of prominent arithmetic models). For example, in the network-retrieval model proposed by Stazyk et al. (1982), it is assumed, much like in the ISM, that operands activate multiples. In this view, activation spreads from each operand node through a network of answer nodes. Each operand activates its associated multiples, and the double activation converging at the correct product identifies the correct node. Campbell and Graham's (1985) network-interference model hypothesized both operand-based activation and problem-based activation from the operand pair. The assumption of problem-based activation is similar to the ISM's assumption of a whole-fact representation. Strengthening of a whole-fact representation provides a hypothetical mechanism for the beneficial effects of practice. Campbell's (1995) revised network-interference model hypothesized problem nodes composed of the two operands: the operation and the answer (see Rickard et al., 1994). Campbell's (1995) proposed network of problem nodes (i.e., operands-operation-answer units), in effect, integrates the operand multiple representation into the whole-fact representation. In this model, both the lure effect and the production of operand multiples as errors occur because of competition from related problems rather than competition from related answers. Our findings, however, do not distinguish between whether whole-fact representation is integrated or independent of the operand multiple representation.

An important new finding in our study was that the lure effect for the two-operand set was amplified when the lure was a correct answer from the two-operand set (i.e., a correct answer to a practiced problem). Both the RT and error analyses demonstrated that the relatively large lure effect for the two-operand set with practiced product lures reflected both decreased performance for practiced productrelated lures and enhanced performance for practiced product-unrelated lures. With respect to greater interference for practiced product-related lures than for unpracticed product-related lures, it appears that strengthening a correct association in the context of one problem (e.g., practicing $4 \times 7=28$ ) increases the difficulty of rejecting a false equation that includes the strengthened association (e.g., 7 and 28 in the context $3 \times 7=28$ ). There could be multiple sources of this effect. It could occur because an illusion of familiarity is produced by presentation of the strengthened components, or because the practiced multiplication fact is strongly activated by presentation of some of its components and this activation creates an experience of familiarity that is mistakenly attributed to the presented equation (see Zbrodoff \& Logan, 1990). Rather than basing the verification decision on familiarity, people may solve a verification equation by retrieving the correct answer and comparing it with the presented answer. In this case, relatively poor performance for practiced productrelated lures could reflect reduced accessibility of the correct answer caused by competition from a strengthened, related fact (Campbell, 1987).
In contrast, no simple explanation for the facilitative effects of practice on unrelated lures presents itself. It cannot be that simply practicing and strengthening the correct association for a product (e.g., $3 \times 7=21$ ) generally makes it easier to reject a false equation involving an unrelated lure (e.g., $4 \times 6=21$ ): The performance advantage for practiced product-unrelated lures relative to unpracticed product-unrelated lures occurred only for the two-operand set; there was no evidence for this effect with zero-operand problems. Therefore, the facilitation effect for practiced product-unrelated lures appears to depend on strengthening the correct association on both sides of a false equation. In this case, both the correct answer to the presented problem and the correct problem to the presented false answer are highly accessible. The facilitation may occur because, for practiced product-unrelated lures, either or both sources of information may be sufficient to reject the equation as false.

\section{CONCLUSIONS}

Our experiment showed that Manly and Spoehr's (1999) evidence for an operand multiples strengthening process was spurious and was caused by a confounding of practiced answers and practiced operands. We found that practiced answers (i.e., strengthened correct associations) produced strong interference in the context of related false equations, whereas multiples of practiced operands that were not practiced answers were subject to RIF. The results extended the RIF phenomenon, which has been studied in the context of word lists, to the domain of memory for number facts. The results also provided direct evidence for the common assumption in models of cognitive arithmetic that numerical facts related to problem operands are covertly processed during retrieval.

\section{REFERENCES}

Anderson, J. R., \& REDER, L. M. (1999). The fan effect: New results and new theories. Journal of Experimental Psychology: General, 128, 186-197.

Anderson, M. C., \& BELL, T. (2001). Forgetting our facts: The role of inhibitory processes in the loss of propositional knowledge. Journal of Experimental Psychology: General, 130, 544-570.

Anderson, M. C., BJORK, R. A., \& BJORK, E. L. (1994). Remembering can cause forgetting: Retrieval dynamics in long-term memory. Journal of Experimental Psychology: Learning, Memory, \& Cognition, 20, 1063-1087.

Anderson, M. C., \& MCCulloch, K. C. (1999). Integration as a general boundary condition on retrieval-induced forgetting. Journal of Experimental Psychology: Learning, Memory, \& Cognition, 25, 608-629.

Anderson, M. C., \& Spellman, B. A. (1995). On the status of inhibitory mechanisms in cognition: Memory retrieval as a model case. Psychological Review, 102, 68-100.

ASHCRAFT, M. H. (1992). Cognitive arithmetic: A review of data and theory. Cognition, 44, 75-106.

CAMPBE Ll, J. I. D. (1987). Network interference and mental multiplication. Journal of Experimental Psychology: Learning, Memory, \& Cognition, 13, 109-123.

CAMPBELl, J. I. D. (1995). Mechanisms of simple addition and multiplication: A modified network-interference theory and simulation. Mathematical Cognition, 1, 121-164. 
Campbell, J. I. D., \& Graham, D. J. (1985). Mental multiplication skill: Structure, process and acquisition. Canadian Journal of Psychology, 39, 338-366.

CAMPBell, J. I. D., \& TARLING, D. P. M. (1996). Retrieval processes in arithmetic production and verification. Memory \& Cognition, 24, 156172.

Manly, C. F., \& Spoehr, K. T. (1999). Mental multiplication: Nothing but the facts? Memory \& Cognition, 27, 1087-1096.

Rickard, T. C., Healy, A. F., \& Bourne, L. E. (1994). On the representation of arithmetic facts: Operand order, symbol, and operation transfer effects. Journal of Experimental Psychology: Learning, Memory, \& Cognition, 20, 1139-1153.
StazyK, E. H., Ashcraft, M. H., \& Hamann, M. S. (1982). A network approach to mental multiplication. Journal of Experimental Psychology: Learning, Memory, \& Cognition, 8, 320-335.

VAN SElST, M., \& JOLICÄUR, P. (1994). A solution to the effect of sample size on outlier elimination. Quarterly Journal of Experimental Psychology, 47A, 631-650.

Winkelman, J. H., \& SCHMidT, J. (1974) Associative confusions in mental arithmetic. Journal of Experimental Psychology, 102, 734-736.

ZBrodofF, N. J., \& LogAn, G. D. (1990). On the relation between production and verification tasks in the psychology of simple arithmetic. Journal of Experimental Psychology: Learning, Memory, \& Cognition, 16, 83-97.

\begin{tabular}{|c|c|c|c|c|}
\hline \multicolumn{5}{|c|}{$\begin{array}{l}\text { APPENDIX A } \\
\text { Verification Problems and Lures Used Across Practiced Product-Related, } \\
\text { Unpracticed Product-Related, Practiced Product-Unrelated, and Unpracticed } \\
\text { Product-Unrelated Lure Conditions When the Two-Operand Set Was 2/5/7/8 }\end{array}$} \\
\hline Problems & PRL & URL & PUN & UUN \\
\hline \multicolumn{5}{|c|}{ Two-Operand Set } \\
\hline $2 \times 5=10$ & 14,16 & $15,12,20,18$ & & \\
\hline $7 \times 2=14$ & 10,16 & $12,24,20,28,18$ & 25 & 15,27 \\
\hline $8 \times 2=16$ & 10,14 & $12,24,20,28,18$ & 25 & 15,27 \\
\hline $5 \times 5=25$ & 35 & 15,20 & 16 & $24,28,18,27$ \\
\hline $5 \times 7=35$ & $25,40,49$ & $28,45,42$ & & $24,27,36$ \\
\hline $8 \times 5=40$ & $25,35,56$ & 25,45 & 49 & $28,42,27,36,54$ \\
\hline $7 \times 7=49$ & 35,56 & 42,63 & 40 & $45,36,54$ \\
\hline $7 \times 8=56$ & 40,49 & 42,63 & & 45,54 \\
\hline \multicolumn{5}{|c|}{ One-Operand Set } \\
\hline $3 \times 5=15$ & 10,25 & $12,24,20,18$ & 14,16 & \\
\hline $6 \times 2=12$ & $10,14,16$ & $24,20,18$ & & 15 \\
\hline $8 \times 3=24$ & 16,40 & $15,12,18,27,36$ & $10,14,25,35$ & 20,28 \\
\hline $5 \times 4=20$ & $10,16,25$ & $15,12,24,28$ & 14 & 18,27 \\
\hline $4 \times 7=28$ & $14,16,35,40$ & $24,20,42,36$ & 25 & $15,18,27$ \\
\hline $9 \times 5=45$ & 35,40 & $63,27,36,54$ & 49,56 & 28,42 \\
\hline $6 \times 7=42$ & $35,49,56$ & $28,36,54$ & 40 & 45 \\
\hline $7 \times 9=63$ & 49,56 & 45,54 & & \\
\hline \multicolumn{5}{|c|}{ Zero-Operand Set } \\
\hline $3 \times 4=12$ & & $15,20,18$ & 10,14 & \\
\hline $6 \times 3=18$ & & $15,12,24,27$ & $10,14,16,25$ & 20,28 \\
\hline $4 \times 4=16$ & & $12,24,20$ & 10,14 & 15,18 \\
\hline $4 \times 6=24$ & & $12,20,28,18,36$ & $14,25,35$ & 15,27 \\
\hline $9 \times 3=27$ & & $15,12,24,45,42,18,36$ & $10,14,16,25,35,40$ & 20,28 \\
\hline $9 \times 4=36$ & & $24,20,28,45,18,27,54$ & $25,35,49$ & 42 \\
\hline $6 \times 6=36$ & & 24,42 & $25,35,40$ & $28,45,27$ \\
\hline $6 \times 9=54$ & & $45,42,63,36$ & $40,49,56$ & \\
\hline
\end{tabular}

Note-PRL, practiced product-related lures; URL, unpracticed product-related lures; PUN, practiced product-unrelated lures; UUN, unpracticed product-unrelated lures. 


\section{APPENDIX B}

Verification Problems and Lures Used for Practiced Product-Related, Unpracticed Product-Related, Practiced Product-Unrelated, and Unpracticed Product-Unrelated Lure Conditions When the Two-Operand Set Was 3/4/6/9

\begin{tabular}{|c|c|c|c|c|}
\hline Problems & PRL & URL & PUN & UUN \\
\hline \multicolumn{5}{|c|}{ Two-Operand Set } \\
\hline $3 \times 4=12$ & 18,16 & 15,20 & & 10,14 \\
\hline $6 \times 3=18$ & $12,24,27$ & 15 & 16 & $20,28,10,14,25$ \\
\hline $4 \times 4=16$ & 12,24 & 20 & 18 & $15,10,14$ \\
\hline $4 \times 6=24$ & $12,18,16,36$ & 20,28 & 27 & $15,14,25,35$ \\
\hline $9 \times 3=27$ & $12,18,24,36$ & $15,45,42$ & 16 & $20,28,10,14,25,35,40$ \\
\hline $9 \times 4=36$ & $18,24,27,54$ & $20,28,45,40$ & & $42,25,35,49$ \\
\hline $6 \times 6=36$ & 24 & 42 & 27 & $28,45,25,35,40$ \\
\hline $6 \times 9=54$ & 36 & $45,42,63$ & & $40,49,56$ \\
\hline \multicolumn{5}{|c|}{ One-Operand Set } \\
\hline $3 \times 5=15$ & $12,18,24$ & $20,10,25$ & 16 & 14 \\
\hline $6 \times 2=12$ & $18,16,24$ & $20,10,14$ & & 15 \\
\hline $8 \times 3=24$ & $12,18,16,27,36$ & 15,40 & & $20,28,10,14,25,35$ \\
\hline $5 \times 4=20$ & $12,16,24$ & $15,28,10,25$ & 18,27 & 14 \\
\hline $4 \times 7=28$ & $16,24,36$ & $20,42,14,35,40$ & 18,27 & 15,25 \\
\hline $9 \times 5=45$ & $27,36,54$ & $63,35,40$ & & $28,42,49,56$ \\
\hline $6 \times 7=42$ & 36,54 & $28,35,49,56$ & & 45,40 \\
\hline $7 \times 9=63$ & 54 & $45,49,56$ & & \\
\hline \multicolumn{5}{|c|}{ Zero-Operand Set } \\
\hline $2 \times 5=10$ & & $15,20,14$ & & \\
\hline $7 \times 2=14$ & & $20,28,10$ & 27 & 15,25 \\
\hline $8 \times 2=16$ & & $20,28,10,14$ & 27 & 15,25 \\
\hline $5 \times 5=25$ & & $15,20,35$ & $18,16,24,27$ & 28 \\
\hline $5 \times 7=35$ & & $28,45,42,25,40,49$ & $24,27,36$ & \\
\hline $8 \times 5=40$ & & $45,25,35,56$ & $27,36,54$ & $28,42,49$ \\
\hline $7 \times 7=49$ & & $42,63,35,56$ & 36,54 & 45,40 \\
\hline $7 \times 8=56$ & & $42,63,40,49$ & 54 & 45 \\
\hline
\end{tabular}

Note-PRL, practiced product-related lures; URL, unpracticed product-related lures; PUN, practiced product-unrelated lures; UUN, unpracticed product-unrelated lures.

(Manuscript received April 29, 2003;

revision accepted for publication September 21, 2003.) 